\title{
Management of Acute Superior Mesenteric Artery Occlusion by Thrombolytic Therapy
}

\author{
Shinichi Okamura Hitoshi Fujiwara Teruhisa Sonoyama \\ Toshiya Ochiai Hisashi lkoma Takeshi Kubota \\ Masayoshi Nakanishi Shojiro Kikuchi Daisuke Ichikawa \\ Kazuma Okamoto Chohei Sakakura Yukihito Kokuba \\ Hiroki Taniguchi Eigo Otsuji
}

Department of Surgery, Division of Digestive Surgery, Kyoto Prefectural

University of Medicine, Kyoto, Japan

\section{Key Words}

Superior mesenteric artery · Occlusion · Thrombolysis

\begin{abstract}
Acute occlusion of the superior mesenteric artery (SMA) causes extensive bowel necrosis, resulting in a poor prognosis with an extremely high mortality rate. An 82-year-old woman was admitted to our hospital with the complaint of abdominal pain. She was diagnosed as having acute SMA occlusion by enhanced CT. Five hours from onset, the first thrombolytic therapy with urokinase was performed, but failed to complete thrombolysis and recanalization of peripheral blood flow. An exploratory laparotomy following the first thrombolytic therapy showed a mild ischemic change in the affected intestine and mesentery, but no sign of necrosis. After the laparotomy, local thrombolytic therapy with angiographic evaluation of blood flow at 24, 36 and $48 \mathrm{~h}$ from the first thrombolysis was performed. As a result, the residual thrombus disappeared and all branches of the SMA became well visualized. The patient was discharged well without a second-look operation or major bowel resection. Sequential intermittent thrombolytic therapy with meticulous angiographic evaluation of blood flow is effective for early-stage acute SMA occlusion.
\end{abstract}

\section{Introduction}

Acute occlusion of the superior mesenteric artery (SMA) causes extensive intestinal necrosis due to the difficulty of early diagnosis, resulting in poor prognosis, with a high 
postoperative mortality rate of $65.2 \%$ [1]. Recent reports indicate that selective thrombolytic therapy with intraarterial infusion of urokinase is effective for acute SMA occlusion diagnosed early after onset [2-6]. Early thrombolytic therapy cannot always induce complete thrombolysis, and even if intestinal necrosis is avoided by administration of initial thrombolytic therapy, indications for additional thrombolytic therapy or the method for monitoring intestinal viability during subsequent follow-up have not been established.

In this report, we present a case of acute SMA occlusion diagnosed early after onset that was successfully treated by sequential and intermittent thrombolytic therapy by intraarterial urokinase infusion with angiographic evaluation of blood flow, thereby avoiding intestinal resection.

\section{Case Report}

An 82-year-old woman with a past history of atrial fibrillation was admitted to our hospital, complaining of acute epigastric pain and stool with fresh blood. Physical examination was as follows: blood pressure $140 / 80$, heart rate $70 / \mathrm{min}, \mathrm{O}_{2}$ saturation $97 \%$ (room air), body temperature $37.2^{\circ} \mathrm{C}$. The abdomen was flat and soft, with no apparent tenderness or sign of peritoneal irritation. Laboratory data on admission revealed an elevated WBC count and mildly elevated hepatic enzyme values (WBC 15,400/mm³, CRP 0.05 mg/dl, LDH 502 IU/l, AST 57 IU/l, ALT 21 IU/l, CPK 113 IU/l). Colonoscopy revealed mildly erosive, edematous mucosa, but no obvious bleeding from the ascending colon to the terminal ileum. Abdominal enhanced CT showed a filling defect in the proximal portion of the SMA main trunk, which led to the diagnosis of acute SMA occlusion (fig. 1). Immediately after that diagnosis, $5 \mathrm{~h}$ from onset, selective SMA angiography was performed, showing complete occlusion of the SMA around the first jejunal artery branch. Subsequently, intraarterial bolus infusion of urokinase $(600,000 \mathrm{IU})$ with thrombus suction was performed. As a result, the thrombus decreased in size and peripheral blood flow improved. However, the thrombus remained, and intramural blood flow of the affected intestine was not visualized, suggesting possible necrosis of the affected intestine (fig. 2). No additional thrombolysis was performed because it would have caused shower embolism. Following the first thrombolytic therapy, an exploratory laparotomy was performed. During surgery, a mild ischemic change was observed at the intestinal wall from the jejunum $40 \mathrm{~cm}$ distal from the Treitz ligament to the ascending colon, but no apparent necrosis. Blood flow in the mesentery was well palpable at the central portion of the SMA, while peripheral blood flow was not palpable. The operation was completed without intestinal resection or direct removal of the thrombus from the SMA.

Immediately after the laparotomy, intravenous continuous infusion of heparin was started. $24 \mathrm{~h}$ after the laparotomy, a second angiography was performed via the catheter that remained in place after first angiography, because clinical signs such as abdominal pain suggested the progression of intestinal ischemia. Peripheral blood flow was well visualized, and no signs of intestinal necrosis were observed. Thereafter, thrombolysis with urokinase infusion (240,000 IU) and suction of the residual thrombus were performed. In addition, $36 \mathrm{~h}$ after the laparotomy, a third angiography and thrombolysis (urokinase 240,000 IU) were performed, showing improvement in peripheral blood flow via the gradual development of collateral blood flow. Follow-up carefully monitored the possible onset of shower embolism by the residual thrombus. Intestinal blood flow was well visualized on CT angiography performed $48 \mathrm{~h}$ after laparotomy ( $60 \mathrm{~h}$ after the first thrombolytic therapy). The patient began to take food by mouth on the 6th day after laparotomy and was discharged from our hospital 35 days after laparotomy. She has been free from relapse, receiving anticoagulant therapy with warfarin.

\section{Discussion}

Early diagnosis and treatment contribute to the improvement of therapeutic results for acute SMA occlusion [1]. A delay in diagnosis results in an extremely high mortality rate due to extensive intestinal necrosis; however, successful resection of necrotic intestine causes short bowel syndrome, severely impairing quality of life. Recent reports indicate that enhanced CT is feasible for early diagnosis of acute SMA occlusion. When acute 
SMA occlusion is suspected from symptom, clinical history and physical findings, enhanced CT plays a role not only in identifying the causal portion, but also in determining the subsequent treatment plan [3]. Early thrombolytic therapy is effective for acute SMA occlusion in avoiding extensive intestinal resection. Since the first report by Jamieson et al. [2], reports that support the usefulness of selective thrombolytic therapy via a catheter for angiography have increased [3-6]. The 'golden hour', the time during which the viability of the ischemic intestine can be preserved, varies, dependent on the portion and extent of occlusion [7]. Muneoka et al. [3] studied 23 cases of acute SMA occlusion treated by selective thrombolytic therapy in Japan, and concluded that the golden hour is $5 \mathrm{~h}$ for occlusion of the SMA main trunk and $12 \mathrm{~h}$ for the occlusion of the distal SMA. The goal of thrombolytic therapy is complete thrombolysis, but administration over $48 \mathrm{~h}$ increases the risk of complication [8]. Easy bleeding due to fibrinolysis promoted by urokinase and shower emboli induced by the release of residual thrombi are known to be the major complications associated with thrombolytic therapy with urokinase. Careful monitoring during $24 \mathrm{~h}$ after the completion of thrombolytic therapy is essential, and emergency laparotomy is mandatory if intestinal necrosis is suspected. Urokinase has no influence during surgery as its half-life in blood is only $16 \mathrm{~min}$. In the present case, the thrombus was detected in the SMA main trunk by angiography performed $5 \mathrm{~h}$ from onset. Therefore, judging that we were within the golden hour, we added urokinase infusion and thrombus suction via the angiography catheter. However, the thrombus remained left, and visualization of intramural blood flow was poor, therefore, we selected exploratory laparotomy.

On the other hand, when no apparent intestinal necrosis is observed during exploratory laparotomy, a clinical consensus concerning the indication of additional thrombolytic therapy or optimal methods for evaluation of intestinal blood flow or viability in the subsequent follow-up is not yet established. Even if intestinal necrosis can be avoided, intestinal ischemia would cause perforation or stricture of the affected intestine $[9,10]$. From 1985 to 2007, 45 cases of thrombolytic therapy for acute SMA occlusion were reported in Japan. We studied those 45 cases, which included the present case (fig. 3 ). Of the 45 cases, complete thrombolysis was successful in 28 cases (63.6\%), and 17 cases (36.4\%) received laparotomy following thrombolytic therapy. Among those 17 patients, intestinal resection was performed in 5 cases (11.1\%) due to intestinal necrosis; the remaining 12 cases (26.7\%) without intestinal necrosis at laparotomy were thereafter free from intestinal necrosis. Of those 12 patients, 2 received angiographic reevaluation of blood flow and subsequent second-look operation, 3 received embolectomy, and 6 were carefully observed by laboratory examination and physical findings without any invasive treatments. In the present case, we performed thrombolytic therapy with angiographic evaluation of blood flow, sequentially and intermittently, after exploratory laparotomy, which enabled us not only to accurately evaluate intestinal blood flow or viability, but also to avoid a second-look operation.

In conclusion, sequential and intermittent thrombolytic therapy with meticulous angiographic evaluation of blood flow is effective in the early stage of acute SMA. In particular, it is much less invasive than strategies described in previous reports and enables accurate evaluation of intestinal blood flow or viability over the time course. 


\begin{tabular}{r|l|l|l} 
Case Reports $/ \mathrm{h}$ & $\begin{array}{l}\text { Case Rep Gastroenterol 2009;3:300-305 } \\
\text { D0l: 10.1159/000239293 }\end{array}$ & Published online: September 22, 2009 & $\begin{array}{l}\text { O 2009 S. Karger AG, Basel } \\
\text { ISSN 1662-0631 } \\
\text { www.karger.com/crg }\end{array}$ \\
\hline
\end{tabular}

Fig. 1. Enhanced CT shows a filling defect at the proximal portion of the SMA main trunk. Arrow indicates thrombus.

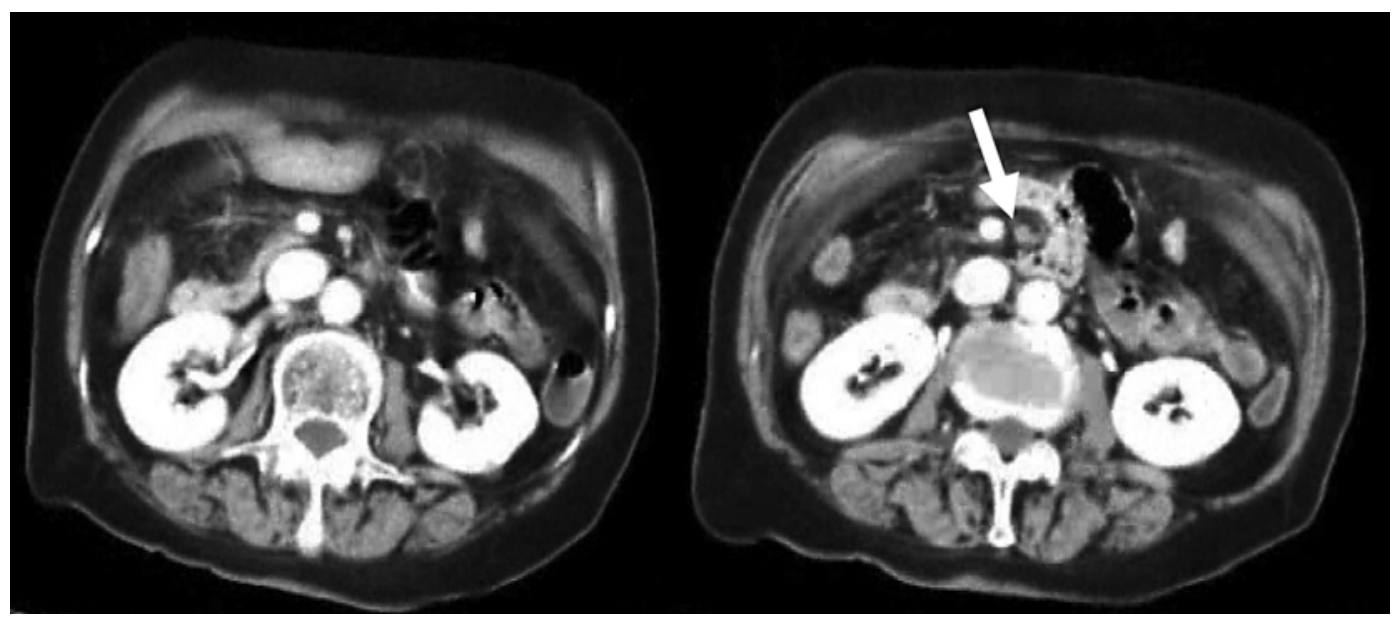

Fig. 2. Selective SMA angiography was performed $5 \mathrm{~h}$ from onset. Before therapy, the SMA was completely occluded by an embolus at the proximal portion (a), and after the bolus infusion of urokinase $(600,000 \mathrm{IU})$ into the SMA, peripheral blood flow was improved. However, the thrombus decreased in size, but remained (b). Asterisks indicate thrombus.

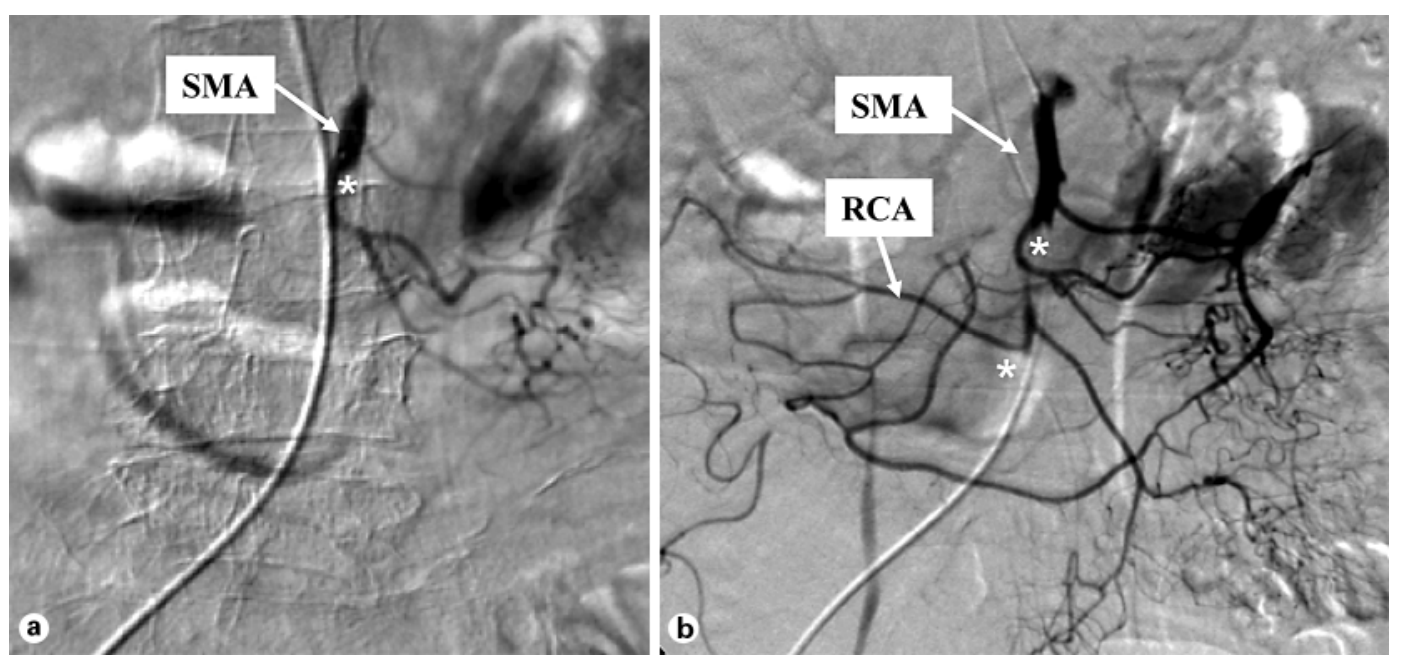


Fig. 3. Forty-five cases of thrombolytic therapy for acute SMA occlusion have been reported in Japan. We studied 45 cases including the present case.

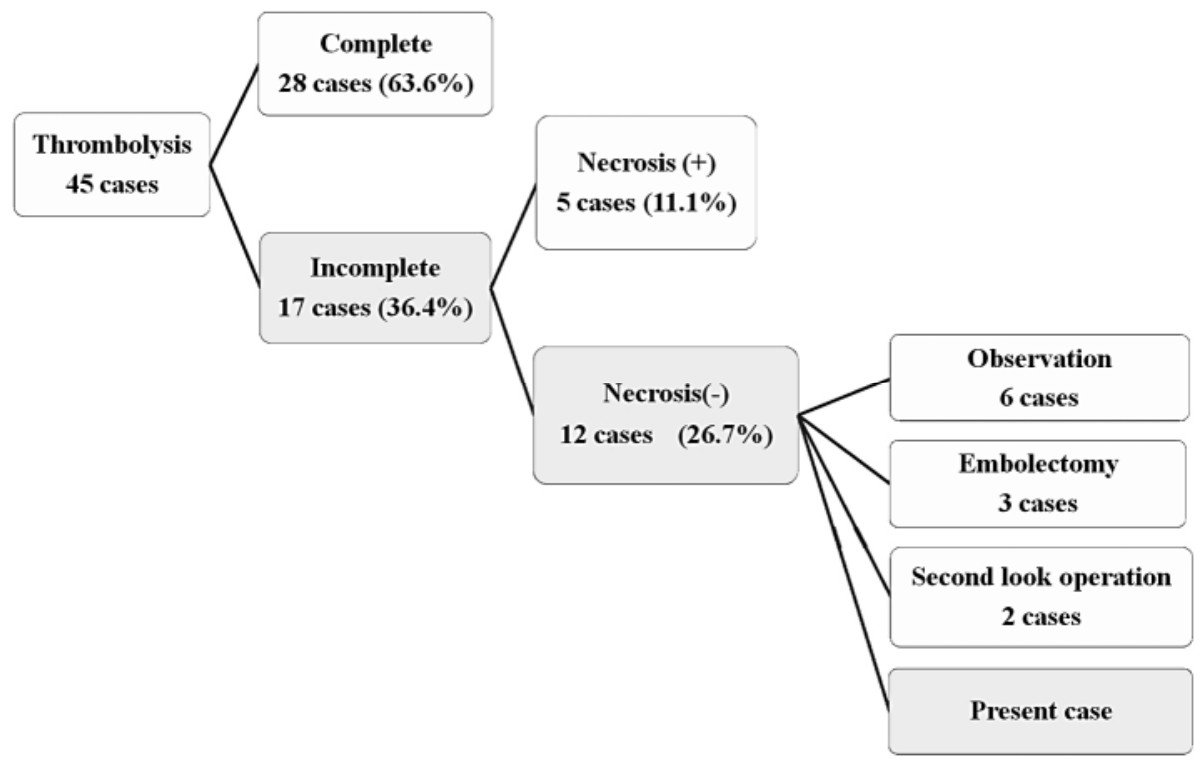




\section{References}

-1 Acosta Merida MA, Marchena Gomez J, Hemmersbach Miller M, Roque Castellano C, Hernandez Romero JM: Identification of risk factors for perioperative mortality in acute mesenteric ischemia. World J Surg 2006;30:15791585.

2 Jamieson AC, Thomas RJ, Cade JF: Lysis of a superior mesenteric artery embolus following local infusion of streptokinase and heparin. Aust N Z J Surg 1979;49:355-356.

3 Muneoka K, Shirai Y, Takagi K, Koyama T: Intraarterial infusion of urokinase for acute superior mesenteric artery occlusion: A report of two cases (in Japanese with English abstract). Jpn J Gastroenterol Surg 2001;34:495-499.

4 Miyazawa T, Ueki K, Wakakuwa R: A case of acute superior mesenteric artery occlusion treated by intraarterial infusion of urokinase and prostaglandin E1 (in Japanese with English abstract). J Jpn Surg Assoc 2004;65:3293-3296.

5 Simo G, Echenagusia AJ, Camunez F, Turegano F, Cabrera A, Urbano J: Superior mesenteric arterial embolism, local fibrinolytic treatment with urokinase. Radiology 1997;204:775-779.

-6 Schoenbaum SW, Pena C, Koenigsberg P, Katzen BT: Superior mesenteric artery embolism, treatment with intraarterial urokinase. J Vasc Intervent Radiol 1992;3:485-490

7 Ottinger LW: The surgical management of acute occlusion of the superior mesenteric artery. Ann Surg 1978;188:721-731.

8 Fujita M: Acute super mesenteric artery occlusion, CT appearance and thrombolytic therapy (in Japanese with English abstract). Jpn J Intervent Radiol 2003;18:22-28.

9 Kato H: A case of superior mesenteric vein thrombosis causing small bowel stricture after conservative treatment (in Japanese with English abstract). J Jpn Surg Assoc 2002;63:1914-1918.

10 Yajima S: A case of acute superior mesenteric artery occlusion, recurrence after intraarterial infusion of urokinase on a month. Jpn Coll Surg 2005;30:907-910. 